\title{
Orthodontics
}

\section{Non-conventional methods for accelerating orthodontic tooth movement. A contemporary overview}

Aslam Alkadhimi Ba.BDent.Sc. (Hon), MFD (RCSI). Orthodontic Specialist Registrar. University College London, Eastman Dental Institute, London and Buckinghamshire Healthcare NHS Trust.

Mohammad Owaise Sharif BDS (Hons), MSc, MOrth RCS Ed, FDS (Ortho) RCS Eng, FHEA. Clinical Lecturer/Honorary Consultant in Orthodontics. University College London, Eastman Dental Institute, London.

\section{Corresponding Author}

Mohammad Owaise Sharif

Department of Orthodontics

Eastman Dental Institute

256 Grays Inn Road

London WC1X 8LD

United Kingdom

Email: mohammad.sharif.16@ucl.ac.uk 


\title{
Orthodontics
}

\section{Non-conventional methods for accelerating orthodontic tooth movement. A contemporary overview}

\author{
Abstract \\ In today's fast paced world, reducing the duration of orthodontic treatment has become a \\ priority for patients seeking treatment. There are now several approaches and devices \\ available that are reported to accelerate orthodontic tooth movement (OTM) and \\ fortunately there has been an increase in the amount of research in this area in recent \\ times.
}

\section{Keywords}

Accelerating, Non-surgical, Orthodontic, Surgical, Tooth movement.

\section{Clinical relevance}

The aim of this paper is to provide an overview of the different non-conventional methods that can be utilised to accelerate OTM and discuss their effectiveness as well as their potential shortcomings.

\section{Objectives}

In recent years, research has evaluated novel approaches to accelerate OTM using a variety of methods. Currently, there are a number of surgical and non-surgical approaches advocated for accelerating OTM. This review explores the effectiveness of these approaches and presents the orthodontic practitioner with a contemporary overview of the topic. 


\section{Introduction}

Orthodontic treatment with fixed appliances can be a lengthy process, comprehensive treatment ranges from 24 to 36 months on average and duration of treatment is one of the main concerns for patients [1]. Approaches to accelerate orthodontic tooth movement (OTM) are therefore welcomed by orthodontists and patients alike. Numerous techniques have evolved over time. Some aim to reduce the treatment duration by accelerating the velocity of OTM, whereas other methods aim to make the mechanical force delivery system more efficient.

Figure 1 illustrates the available surgical and non-surgical methods for accelerating OTM. Surgical techniques on the whole, aim to facilitate tooth movement by inducing a regional acceleratory phenomenon (RAP) [2, 3]. Regional acceleratory phenomenon as described by Harold Frost (1983), is a tissue reaction to noxious stimuli that increases healing capacity. However, the use of surgical approaches is limited given the invasiveness of some techniques and the low quality of evidence to support their use. In light of this, the majority of research has focused on non-surgical approaches these can be further sub divided into physical, biological and/or mechanical methods.

The aim of this paper is to provide the orthodontic practitioner with an overview of the available methods for accelerating OTM and to summarise the available evidence for their use. 


\section{1- Non-surgical approaches to accelerating OTM}

\section{Mechanical}

\section{- Self-ligating brackets}

\section{Proposed method of action}

Since their introduction, self-ligating brackets (SLB) have been reported to reduce friction and lead to a decrease in treatment duration. There are two main SLB designs: active SLBs (can apply force on the archwire, owing to the spring clip) and passive SLBs (do not exert active force and do not encroach on the slot lumen). Examples of self-ligating systems include Damon ${ }^{\circledR}$ (Figure 2), Speed ${ }^{\mathrm{TM}}$, or In-Ovation ${ }^{\circledR}$. The potential benefits of SLBs include; reduced treatment time (reported to be due to the reduced friction between the archwire and bracket slots), reduced plaque build-up (given that there are no elastic modules which can make plaque removal more difficult) and reduced chair side time (reported to be because ligation of the archwire does not involve the transfer of modules).

\section{Supporting evidence}

The first published clinical studies (retrospective design) on treatment efficiency became available in 2001 and concluded that the use of SLBs resulted in shorter treatment duration $[4,5]$. However, subsequently prospective clinical trials and a number of systematic reviews using randomised controlled trials have concluded that there is no difference between conventional brackets and self-ligating brackets with regards to treatment duration, efficiency of space closure, speed of alignment or transverse changes [6-12].

\section{Clinical relevance}

A recent meta-analysis concluded that there was no difference between SLB and conventional brackets in terms of rate of space closure (MD $0.13 \mathrm{~mm}, 95 \% \mathrm{CI}-0.09$ to 
0.35) or efficiency of alignment (MD -4.69 days, 95\% CI -22.28 to 12.91[12]. However, this meta-analysis did conclude that active SLBs appear to be more efficient for alignment compared to passive SLBs and conventional brackets (MD -10.24 days, 95\% confidence interval (CI) -17.68 to -2.80$)$. Given the confidence interval, this is likely to be a clinically insignificant difference.

\section{- Customised fixed labial appliances}

\section{Proposed method of action}

Brackets customised to individual tooth surfaces are created and bonded indirectly using placement guides. Examples include SureSmile ${ }^{\mathrm{TM}}$ (OraMetrix, Richardson, TX) which uses 3D scans to provide robotically-bent wires to move the teeth into their desired positions (the system compensates for errors in bracket placement. As opposed to other systems, SureSmile ${ }^{\mathrm{TM}}$ customisation takes place in the finishing stages of orthodontic treatment, i.e. by customising the archwires and not the brackets. In contrast, in some systems, for example Insignia ${ }^{\mathrm{TM}}$ (Ormco Corporation, Orange CA), bracket bases are standard; slots are custom created to produce the desired tooth movement via arch wire progression to a straight final arch wire. The aim of the aforementioned systems being to increase precision and eliminate human error in archwire bending and bracket placement. The proposed advantages of such systems include:

- an increased accuracy in bracket positioning and thereby a reduced need for bracket repositioning

- minimal dependence on wire bending

\section{Supporting evidence}

To date, there is only one published randomised controlled trial (RCT) investigating the difference in treatment duration between a customised fixed appliance system (Insignia) 
and a non-customised system (Damon Q). The authors concluded that "the customized group had more loose brackets, a longer planning time, and more complaints $(\mathrm{P}<0.05)$. The customized orthodontic system was not associated with significantly reduced treatment duration, and treatment quality was comparable between the 2 systems" [13]. Currently, there are no other published trials on regarding this topic. The existing knowledge around the efficiency of customised labial appliances has consisted mostly of expert opinion, case reports and a retrospective study.

\section{Clinical relevance}

Owing to the limitations regarding the available knowledge base discussed above, at present, no conclusion can be made regarding the effectiveness of customised labial appliances in terms of treatment efficiency and speed of alignment given the lack of evidence in the literature.

\section{- Microvibration}

Proposed method of action

It is reported that vibration leads to stimulation of cell differentiation and maturation, thereby increasing the rate of bone remodelling and turnover. From that perspective, the effect of vibratory appliances appears to be linked to local injury (i.e. inducing microfractures in the alveolar bone). An example of this approach is the AcceleDent ${ }^{\mathrm{TM}}$ device (OrthoAccel Technologies, Inc. Houston, TX). AcceleDent ${ }^{\mathrm{TM}}$ was first introduced in 2009 (Figure 3). It provides low-frequency vibratory forces $(30 \mathrm{~Hz})$ which produce around 25 grams of force with the view to stimulating cell differentiation and maturation thereby accelerating bone remodelling and hence tooth movement [6]. 


\section{Supporting evidence}

A systematic review published in 2017 [14] assessing the effectiveness of vibrational stimulus that included eight prospective clinical trials with an overall sample of 305 patients concluded that there is "weak evidence indicates that vibrational stimulus is effective for accelerating canine retraction but not for alignment". However, the heterogeneity in methodology and non-comparability of outcome measures utilised in the studies prevented a quantitative synthesis from being performed.

\section{Clinical relevance}

The general consensus in the literature at present is that microvibration does not cause clinically significant increase of OTM in terms of initial alignment phase or rate space closure $[6,15-17]$

\section{Biological}

\section{- Pharmacological agents/ Exogenous molecules}

\section{Proposed method of action}

Pharmacological agents have been used in an attempt to alter the biological response to orthodontic force [6]. The vast majority of the data comes from animal studies rather than human studies and although an insight to their effects is provided, the results must be interpreted with caution.

\section{Supporting evidence}

A recent meta-analysis of twenty-seven animal studies found that the rate of orthodontic tooth movement increases after the administration of diazepam, Vitamin $\mathrm{C}$ and pantoprazole, while simvastatin, atorvastatin, calcium compounds, strontium ranelate, propranolol, losartan, famotidine, cetirizine, and metformin decreased the rate of orthodontic tooth movement [18]. Additionally, there are a number pharmacogical agents 
that may reduce the rate of OTM (e.g. drugs blocking the action of prostaglandins, such as aspirin and NSAID's), common pharmacological agents and systemic factors and their effect on OTM have been summarised in Table 2.

\section{Clinical relevance}

The practical use of these exogenous molecules/medications is limited because of the need for regular administration (as frequently as every week) and the anxiety discomfort associated with injections. 


\section{Physical}

\section{- Photobiomodulation}

\section{Proposed method of action}

Photobiomodulation, also known as low-level light therapy (LLLT) utilises low energy lasers or light-emitting diodes (LED) in an attempt to modify cellular biology. The theory is that exposure to light in the red to near-infrared range $(600-1000 \mathrm{~nm})$ induces a photochemical reaction at the cellular level, light energy is absorbed by the cellular photoreceptors and converted into adenosine triphosphate by mitochondria [6]. This subsequently increases the cellular activities such as DNA, RNA, and protein synthesis thereby potentially accelerating OTM. It is suggested that a 10 seconds exposure to a diode laser emitting light for $20 \mathrm{~mW}$ once a week is required to induce potentially clinical effect [19].

\section{Supporting evidence}

The first published randomised controlled trial investigating the effect of LLLT on OTM was reported in 2004 [19]. This was a split mouth trial and concluded that LLLT does accelerate the rate of canine retraction. However, the rate of acceleration was clinically insignificant, after 60 days, the canine retraction was $4.39 \pm 0.27 \mathrm{~mm}$ for the intervention group and 3.30 $\pm 0.24 \mathrm{~mm}$ for the control group. Since 2004, several RCTs have been carried out. The most recent trial (split mouth design investigating the rate of canine retraction in premolar extractions cases) found that LLLT may accelerate OTM after 10 irradiations [20]. The this study, the canines moved $1.1 \mathrm{~mm}$ more on the intervention side than the control side after 84 days. However, the reduction in treatment time was not clinically significant. A recent meta-analysis of six RCTs suggests that the application of LLLT may accelerate OTM [21]. Orthodontic tooth movement was statistically increased in the LLLT group 
compared with the control group in 21 days. The authors concluded that "the LLLT can speed up the rate of tooth movement of human canine and consequently decrease the treatment time". The results showed that the orthodontic movement of canine was statistically increased in the LLLT group compared with the control group in 21 days (MD: 0.74mm; 95\%CI: $0.17-1.31 ; \mathrm{P}=0.01$ ) and 4.5 months (MD: $1.53 \mathrm{~mm} ; 95 \% \mathrm{CI}: 0.92-2.14$; $\mathrm{P}<0.001)$. However, these changes are unlikely to be clinically significant, also, given the small sample sizes of the included RCTs, the effect of LLLT on rate of OTM should be interpreted with caution.

\section{Clinical relevance}

In light of the above, there is limited evidence suggesting that there may be an enhanced rate of OTM with the application of LLLT. However, this is an area that requires further research before clinical recommendations can be made.

\section{- Electromagnetic fields}

Proposed method of action

The proposed mechanism of action is that electromagnetic fields affect the activity of intracellular cyclic adenosine monophosphate and cyclic guanesine monophosphate. This may subsequently lead to an acceleration of bone remodelling and hence OTM. A circuit and watch battery is used to generate approximately $1 \mathrm{~Hz}$ of electric current in a removable appliance. 


\section{Supporting evidence}

A single trial (non-randomised prospective design) assessing the effect of electromagnetic fields on OTM was identified, this trial showed an increase in OTM of $0.3 \mathrm{~mm} / \mathrm{month}$ in relation to canine retraction and space closure [22].

\section{Clinical relevance}

Given the lack of evidence associated with the application of electromagnetic fields and direct electric currents, its clinical use cannot be recommended at present. 


\section{2-Surgical approaches to accelerating OTM}

\section{- Micro-osteoperforation}

Proposed method of action

Micro-osteoperforation (MOP) refers to localised bone trauma in the region where acceleration of OTM is required. Trauma to bone subsequently induces RAP. Microosteoperforation is a relatively minimally invasive procedure, as there is no need to raise a full thickness flap or to make separate soft tissue incisions prior to the osteoperforation. Propel $^{\mathrm{Tm}}$ is an example of one device that can be used (Figure 4). It has a pointed surgical stainless steel tip of $1.6 \mathrm{~mm}$ in diameter at its widest aspect and a usable length of up to 7.0 $\mathrm{mm}$. It is used to create small MOPs (usually 3) in the extraction spaces directly through the gingival tissue into bone.

\section{Supporting evidence}

There are currently four randomised controlled trials investigating the effectiveness of this method. The findings of these trials are summarised in Table 1.

\section{Clinical relevance}

The findings from these four trials on average demonstrate reduction in time for canine retraction by up to 3 months in MOP groups. However, despite the statistically significant increase in rate of canine retraction, at present the authors were unable to identify any published studies evaluating the effects of MOPs over the whole course of orthodontic treatment.

\section{- Piezocision}


This procedure adopts the principles of MOP in terms of mechanism of action, however, it is more invasive. It involves creating incisions in the buccal/labial gingiva parallel to long axes of teeth followed by incisions in the buccal cortical plates using a Piezo surgical knife under local anaesthetic.

\section{Supporting evidence}

Recently published systematic reviews based on RCTs have concluded that there is weak evidence to suggest that this procedure is a safe adjunct to accelerate OTM and is up to two times faster than those of a conventional method [23, 24]. However, in one of the systematic reviews only 2 RCTs investigating effects of piezocision were included, both with very small sample sizes (10 patients and 20 patients). These results therefore need to be interpreted with caution as the sample size of included studies was small and might not be representative.

\section{Clinical relevance}

Given the limitations of the existing evidence, further high quality clinical trials are needed to determine the long-term effects and optimal protocol for piezocision prior to drawing more definitive conclusions. Well-designed RCTs are required to confirm the rate of acceleration, risk-benefit ratio, patient perception, long-term follow-up and relapse after corticotomy and piezocision.

\section{- Corticotomy}

\section{Proposed method of action}

A corticotomy is defined as a surgical procedure whereby the cortical bone is cut, perforated, or mechanically altered. Kole was the first to describe modern-day corticotomy-facilitated 
orthodontics. He used the term "bony block" to describe the suspected mode of movement after corticotomy [25]. Selective alveolar corticotomy can be used in most cases in which traditional fixed orthodontic therapy is used. Unlike MOP and piezocision, which penetrate the cortical bone through the overlying tissue, corticotomy requires raising a full thickness mucoperiosteal flap. The procedure is usually carried out under local anaesthesia, vertical incisions are made between the roots of the teeth horizontally $2-3 \mathrm{~mm}$ above the apices (in order not to damage nerve and blood supply). Trauma to bone subsequently induces RAP and may therefore accelerate OTM.

\section{Supporting evidence}

A recent systematic review concluded that this technique may be effective in accelerating OTM. The authors concluded that "Corticotomy-facilitated orthodontics resulted in decreased treatment time. Few complications and low morbidity were found. More solid evidence-based research is required to support these results" [26].

Another systematic review concluded that corticotomy resulted in greater acceleration of OTM than did conventional techniques. The rate of orthodontic tooth movement in corticotomy varied with an increase of 1.5 to 4 times that of the conventional rate of tooth movement, this varied depending on the surgical methods used [24].

\section{Clinical relevance}

There certainly has been a growing interest in the use of corticotomies as an adjunct to orthodontic treatment due to a deeper understanding of its effects and the emerging evidence base. However, this technique is more invasive that those previously described, is more costly as it often needs to be performed by an oral surgeon or periodontist and arguably has a higher risk of morbidity compared with MOP and piezocision. 


\section{- Segmental osteotomy}

Proposed method of action

A segmental osteotomy may be performed by either distraction of periodontal ligament (involves reduction of the interseptal bone distal to the tooth to be retracted) or distraction of dento-alveolus (this involves a larger osteotomy to fully mobilise the dentoalveolar segment surrounding the tooth to be retracted). These procedures are limited to single tooth retraction, usually maxillary canine retraction after premolar extraction. Again, trauma to bone from this procedure subsequently induces RAP and hence may accelerate OTM.

\section{Supporting evidence}

The application of this technique is very limited owing to the invasiveness of the surgeries. There is currently a low level of evidence to support its clinical use [27].

\section{Clinical relevance}

In the studies reviewed, there were contradictory results regarding of the pulp vitality of the retracted canines. Liou and colleagues in 1998 reported 9 out of 26 teeth showed positive vitality [28], while other workers reported that 7 out of 20 showed positive vitality after the sixth month of retraction [29]. Therefore, there are still some uncertainties regarding this technique and there is a need for more research with additional attention paid to adverse effects and cost-benefit ratio.

\section{- $\quad$ Surgery-first}

\section{Proposed method of action}

This concept was first introduced in Japan in 2009 [30]. A 'surgery-first' approach preceding orthodontic treatment has been suggested in cases requiring orthognathic correction as part 
of the overall comprehensive orthodontic/orthognathic treatment [30]. Traditionally, prior to orthognathic treatment, orthodontic treatment is initiated to prepare the occlusion for surgery. However, the surgery-first approach in its purest form involves performing orthognathic prior to orthodontic tooth movement. The types of cases best suited to this approach meet certain criteria;

- patients presenting with mild or no crowding,

- a flat to mild curve of Spee,

- normal to mild proclination/ retroclination of incisors, and

- minimal or no transverse discrepancies.

The theory is that a region acceleratory phenomenon is initiated by the orthognathic surgery which allows for the subsequent OTM to be accelerated.

\section{Supporting evidence}

This approach has not been the subject of a prospective randomised trial [6] and therefore definitive conclusions cannot be drawn. A recent meta-analysis (including 12 observational studies: 498 participants) found that the pooled estimate suggested that the surgery-first group manifested less postoperative stability (moderate heterogeneity) than traditional approach group [31]. Therefore, patient screening and treatment planning should be reviewed carefully to compensate for possible postoperative relapse when adopting surgeryfirst approach.

\section{Clinical significance}

Further research and work relating to standardised treatment protocols is required prior to the potential wider implementation of this approach. 


\section{Discussion}

In recent times, there has been an increase in the number of approaches aimed at accelerating OTM. In this article, we have explored the majority of proposed surgical and non-surgical approaches proposed. A Cochrane review (2015), summarises the effectiveness of the nonsurgical approaches as follows: "there is very little clinical research concerning the effectiveness of non-surgical interventions to accelerate orthodontic treatment. The available evidence is of very low quality and so it is not possible to determine if there is a positive effect of non-surgical adjunctive interventions to accelerate tooth movement" [32]. The updated literature in this current review article continues to support this view.

Surgical approaches to accelerate OTM are more invasive in nature and thus less widely applied. A Cochrane review (2015), summarises the effectiveness of the surgical approaches as follows: "there is limited research concerning the effectiveness of surgical interventions to accelerate orthodontic treatment, with no studies directly assessing our prespecified primary outcome. The available evidence is of low quality, which indicates that further research is likely to change the estimate of the effect. Based on measured outcomes in the short-term, these procedures do appear to show promise as a means of accelerating tooth movement. It is therefore possible that these procedures may prove useful" [33].

A number of studies have been published subsequent to the aforementioned Cochrane reviews and the authors of this current review have attempted to incorporate this additional evidence. Despite the availability of new trials the conclusions remain largely unchanged. 


\section{Conclusions}

At present, there is insufficient evidence to support the use of the majority of approaches reported to accelerate OTM. Non-surgical approaches can be difficult to apply in everyday practice due to the use of expensive and specialized equipment and the need for regular and repeated administration of the intervention. The evidence to support surgical approaches to accelerating OTM is limited and they are associated with significant invasiveness, exposing the patient to additional stress and postoperative pain. Of the surgical approaches reviewed, MOP seems to be most promising; however, more clinical trials are needed before clinical recommendations can be made.

\section{Declarations}

- Ethics approval and consent to participate: N/A.

- Consent for publication: consent obtained for figures 2-4.

- Availability of data and material: Literature search available.

- Competing interests: N/A.

- Funding: Mohammad Owaise Sharif was awarded the Royal College of Surgeons of England Faculty of Dental Surgery $70^{\text {th }}$ Anniversary Research Fellowship.

- Authors' contributions: Both authors prepared this manuscript.

- Acknowledgements: N/A.

- No potential conflict of interest was reported by the author(s). 


\section{Figures and Tables}

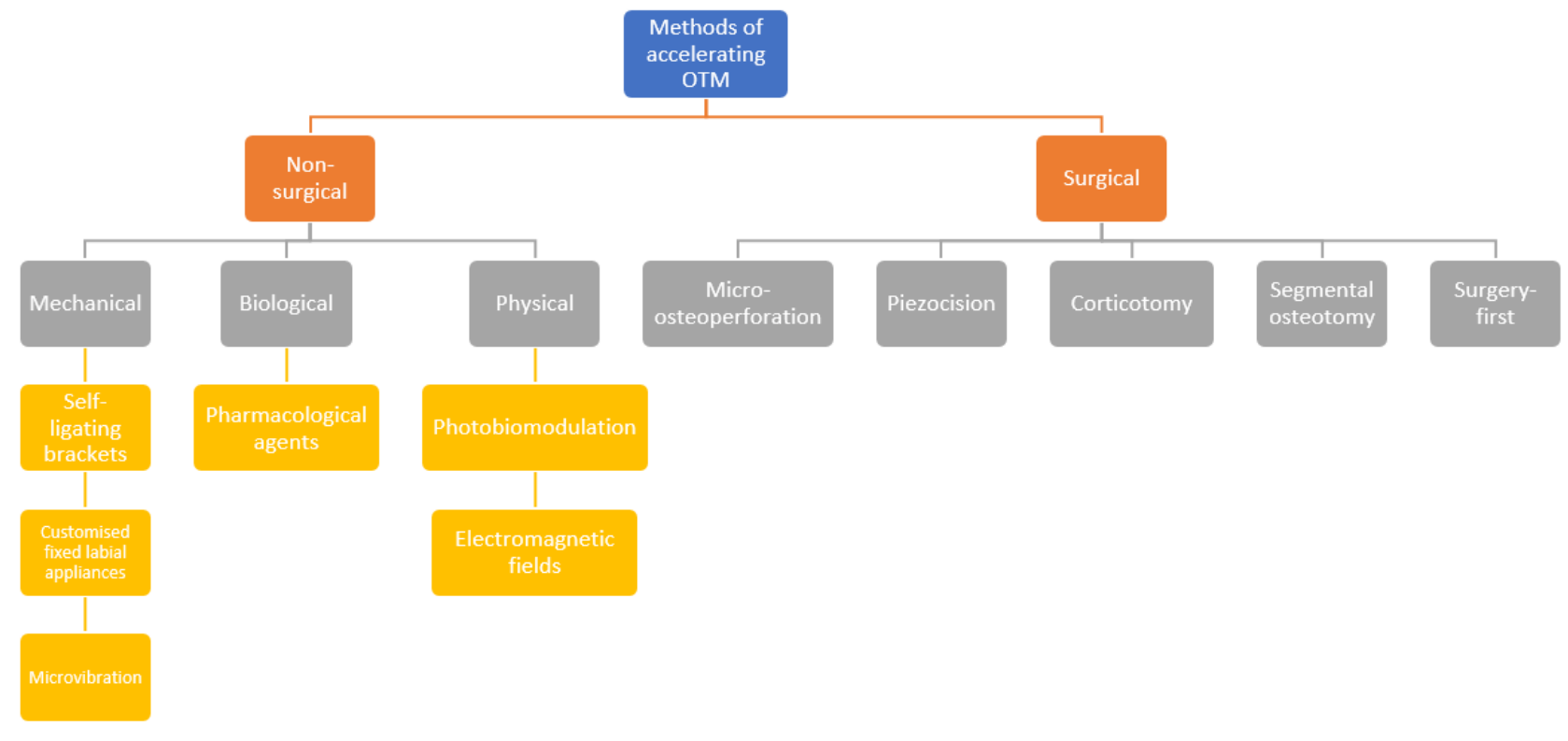

Figure 1. An overview of methods for accelerating orthodontic tooth movement.

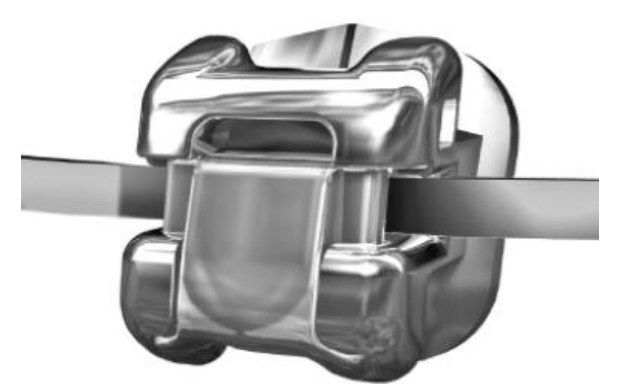

Figure 2. Example of a self-ligating bracket (Damon $\left.{ }^{\mathrm{TM}}\right)$.

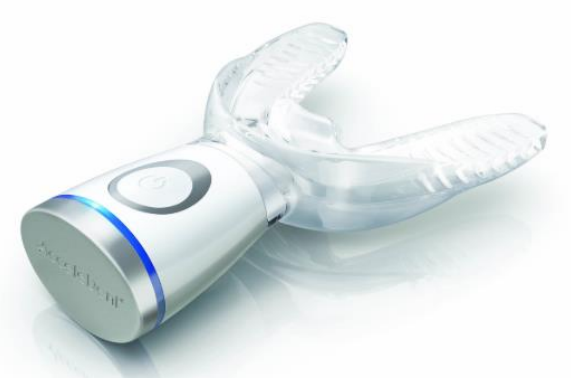

Figure 3. AcceleDent ${ }^{\circledR}$ Optima ${ }^{\mathrm{TM}}$. A hands-free portable device consisting of an activator unit and a removable thermoplastic occlusal wafer that the patient bites onto. The manufacturer recommends the device to be used for 20 minutes per day to deliver 25 grams of force during active orthodontic treatment. 


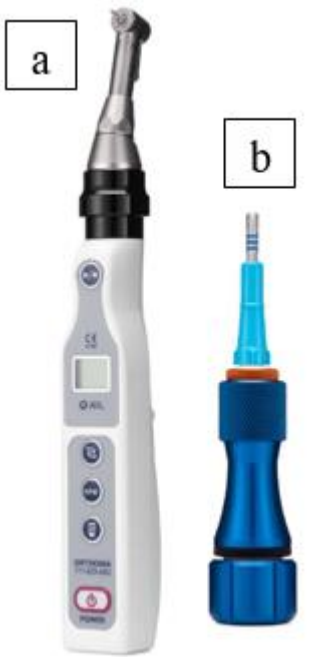

Figure 4. Examples of MOPs devices by Propel $^{\circledR}$ Orthodontics, a) Excellerator ${ }^{\circledR}$ PT and b) Excellerator $^{\circledR}$ RT. 


\begin{tabular}{|c|c|c|}
\hline Drugs & $\begin{array}{l}\text { Effects on bone } \\
\text { metabolism }\end{array}$ & Effects on tooth movement \\
\hline \multicolumn{3}{|c|}{ Non-Steroidal Anti-Inflammatory Drugs (NSAIDs) } \\
\hline Aspirin & Decrease bone resorption & Decrease tooth movement \\
\hline Diclofenac & Decrease bone resorption & Decrease tooth movement \\
\hline Ibuprofen & Decrease bone resorption & Decrease tooth movement \\
\hline Indomethacin & Decrease bone resorption & Decrease tooth movement \\
\hline Celecoxib & Decrease bone resorption & No influence \\
\hline \multicolumn{3}{|c|}{ Acetaminophen analgesics } \\
\hline Paracetamol & Unproven & No influence \\
\hline \multicolumn{3}{|c|}{ Miscellaneous drugs } \\
\hline Prostaglandins & Stimulate bone resorption & Increase tooth movement \\
\hline Corticosteroids & Increase bone resorption & Increase tooth movement \\
\hline Leukotrienes & Stimulate bone resorption & Increase tooth movement \\
\hline Bisphosphonates & Decrease bone resorption & Decrease tooth movement \\
\hline Interleukin Antagonist & Reduced bone remodelling & Decrease tooth movement \\
\hline Fluorides & Inhibit osteoclastic activity & Decrease tooth movement \\
\hline \multicolumn{3}{|c|}{ Systemic factors } \\
\hline Parathyroid hormone & Increase bone resorption & Increase tooth movement \\
\hline Thyroid hormone & $\begin{array}{l}\text { Increase rate of bone } \\
\text { remodelling }\end{array}$ & Increase tooth movement \\
\hline Vitamin D & $\begin{array}{l}\text { Increase rate of bone } \\
\text { remodelling }\end{array}$ & Increase tooth movement \\
\hline Relaxin & Increase bone resorption & Increase tooth movement \\
\hline Oestrogen & Decrease bone resorption & Decrease tooth movement \\
\hline Calcitonin & Inhibit bone resorption & Decrease tooth movement \\
\hline
\end{tabular}

Table 2. Effects of common medications and systemic factors on the rate of tooth movement [38-42]. 


\section{References}

1. Mavreas, D. and A.E. Athanasiou, Factors affecting the duration of orthodontic treatment: $a$ systematic review. Eur J Orthod, 2008. 30(4): p. 386-95.

2. Gkantidis, N., et al., Effectiveness of non-conventional methods for accelerated orthodontic tooth movement: a systematic review and meta-analysis. Journal of Dentistry, 2014. 42(10): p. 1300-19.

3. Fleming, P.S., Accelerating orthodontic tooth movement using surgical and non-surgical approaches. Evidence-Based Dentistry, 2014. 15(4): p. 114-5.

4. Eberting, J.J., S.R. Straja, and O.C. Tuncay, Treatment time, outcome, and patient satisfaction comparisons of Damon and conventional brackets. Clinical Orthodontics \& Research, 2001. 4(4): p. 228-34.

5. Harradine, N.W., Self-ligating brackets and treatment efficiency. Clin Orthod Res, 2001. 4(4): p. 2207.

6. Miles, P., Accelerated orthodontic treatment - what's the evidence? Australian Dental Journal, 2017. 62 Suppl 1: p. 63-70.

7. Celar, A., et al., Systematic review on self-ligating vs. conventional brackets: initial pain, number of visits, treatment time. J Orofac Orthop, 2013. 74(1): p. 40-51.

8. Fleming, P.S. and A. Johal, Self-ligating brackets in orthodontics. A systematic review. Angle Orthod, 2010. 80(3): p. 575-84.

9. Papageorgiou, S.N., et al., Clinical effects of pre-adjusted edgewise orthodontic brackets: a systematic review and meta-analysis. Eur J Orthod, 2014. 36(3): p. 350-63.

10. Dehbi, H., et al., Therapeutic efficacy of self-ligating brackets: A systematic review. Int Orthod, 2017. 15(3): p. 297-311.

11. Yang, X., et al., Differences between active and passive self-ligating brackets for orthodontic treatment : Systematic review and meta-analysis based on randomized clinical trials. Journal of Orofacial Orthopedics, 2017. 78(2): p. 121-128.

12. Yang, X., et al., Transversal changes, space closure, and efficiency of conventional and self-ligating appliances : A quantitative systematic review. Journal of Orofacial Orthopedics, 2018. 79(1): p. 1-10.

13. Penning, E.W., et al., Orthodontics with Customized versus Noncustomized Appliances: A Randomized Controlled Clinical Trial. J Dent Res, 2017. 96(13): p. 1498-1504.

14. Jing, D., et al., The effectiveness of vibrational stimulus to accelerate orthodontic tooth movement: a systematic review. BMC Oral Health, 2017. 17(1): p. 143.

15. DiBiase, A.T., et al., Effect of supplemental vibrational force on orthodontically induced inflammatory root resorption: A multicenter randomized clinical trial. Am J Orthod Dentofacial Orthop, 2016. 150(6): p. 918-927.

16. DiBiase, A.T., et al., Effects of supplemental vibrational force on space closure, treatment duration, and occlusal outcome: A multicenter randomized clinical trial. Am J Orthod Dentofacial Orthop, 2018. 153(4): p. 469-480.e4.

17. Katchooi, M., et al., Effect of supplemental vibration on orthodontic treatment with aligners: $A$ randomized trial. American Journal of Orthodontics \& Dentofacial Orthopedics, 2018. 153(3): p. 336346.

18. Makrygiannakis, M.A., E.G. Kaklamanos, and A.E. Athanasiou, Effects of systemic medication on root resorption associated with orthodontic tooth movement: a systematic review of animal studies. Eur J Orthod, 2018.

19. Cruz, D.R., et al., Effects of low-intensity laser therapy on the orthodontic movement velocity of human teeth: a preliminary study. Lasers Surg Med, 2004. 35(2): p. 117-20.

20. Arumughan, S., et al., A Comparison of the Rate of Retraction with Low-level Laser Therapy and Conventional Retraction Technique. Contemporary Clinical Dentistry, 2018. 9(2): p. 260-266.

21. Imani, M.M., et al., Effect of Low-level Laser Therapy on Orthodontic Movement of Human Canine: a Systematic Review and Meta-analysis of Randomized Clinical Trials. Acta Informatica Medica, 2018. 26(2): p. 139-143. 
22. Showkatbakhsh, R., A. Jamilian, and M. Showkatbakhsh, The effect of pulsed electromagnetic fields on the acceleration of tooth movement. World Journal of Orthodontics, 2010. 11(4): p. e52-6.

23. Hoffmann, S., et al., Influence of piezotomy and osteoperforation of the alveolar process on the rate of orthodontic tooth movement: a systematic review. Journal of Orofacial Orthopedics, 2017. 78(4): p. 301-311.

24. Viwattanatipa, N. and S. Charnchairerk, The effectiveness of corticotomy and piezocision on canine retraction: A systematic review. Korean J Orthod, 2018. 48(3): p. 200-211.

25. Kole, H., Surgical operations on the alveolar ridge to correct occlusal abnormalities. Oral Surg Oral Med Oral Pathol, 1959. 12(5): p. 515-29 concl.

26. Gil, A.P.S., et al., Alveolar corticotomies for accelerated orthodontics: A systematic review. J Craniomaxillofac Surg, 2018. 46(3): p. 438-445.

27. Alfawal, A.M., et al., Effectiveness of minimally invasive surgical procedures in the acceleration of tooth movement: a systematic review and meta-analysis. Progress in Orthodontics, 2016. 17(1): p. 33.

28. Liou, E.J., J.W. Polley, and A.A. Figueroa, Distraction osteogenesis: the effects of orthodontic tooth movement on distracted mandibular bone. J Craniofac Surg, 1998. 9(6): p. 564-71.

29. Sukurica, Y., et al., Rapid canine distalization through segmental alveolar distraction osteogenesis. Angle Orthod, 2007. 77(2): p. 226-36.

30. Nagasaka, H., et al., "Surgery first" skeletal Class III correction using the Skeletal Anchorage System. J Clin Orthod, 2009. 43(2): p. 97-105.

31. Wei, H., et al., Surgery-first/early-orthognathic approach may yield poorer postoperative stability than conventional orthodontics-first approach: a systematic review and meta-analysis. Oral Surg Oral Med Oral Pathol Oral Radiol, 2018. 126(2): p. 107-116.

32. El-Angbawi, A., et al., Non-surgical adjunctive interventions for accelerating tooth movement in patients undergoing fixed orthodontic treatment. Cochrane Database of Systematic Reviews, 2015(11): p. CD010887.

33. Fleming, P.S., et al., Surgical adjunctive procedures for accelerating orthodontic treatment. Cochrane Database Syst Rev, 2015(6): p. Cd010572.

34. Alkebsi, A., et al., Three-dimensional assessment of the effect of micro-osteoperforations on the rate of tooth movement during canine retraction in adults with Class II malocclusion: A randomized controlled clinical trial. Am J Orthod Dentofacial Orthop, 2018. 153(6): p. 771-785.

35. Sivarajan, S., et al., Mini-implant supported canine retraction with micro-osteoperforation: A splitmouth exploratory randomized clinical trial. Angle Orthod, 2018.

36. Attri, S., et al., Comparison of rate of tooth movement and pain perception during accelerated tooth movement associated with conventional fixed appliances with micro-osteoperforations - a randomised controlled trial. Journal of Orthodontics, 2018: p. 1-9.

37. Alikhani, M., et al., Effect of micro-osteoperforations on the rate of tooth movement. Am J Orthod Dentofacial Orthop, 2013. 144(5): p. 639-48.

38. Diravidamani, K., S.K. Sivalingam, and V. Agarwal, Drugs influencing orthodontic tooth movement: An overall review. J Pharm Bioallied Sci, 2012. 4(Suppl 2): p. S299-303.

39. Fernandez-Gonzalez, F.J., et al., Experimental evidence of pharmacological management of anchorage in Orthodontics: A systematic review. Dental Press J Orthod, 2015. 20(5): p. 58-65.

40. Krishnan, V. and Z. Davidovitch, The effect of drugs on orthodontic tooth movement. Orthod Craniofac Res, 2006. 9(4): p. 163-71.

41. Cadenas de Llano-Perula, M., et al., Effectiveness of Biology-Based Methods for Inhibiting Orthodontic Tooth Movement. A Systematic Review. J Clin Pediatr Dent, 2017. 41(6): p. 494-502.

42. Bartzela, T., et al., Medication effects on the rate of orthodontic tooth movement: a systematic literature review. Am J Orthod Dentofacial Orthop, 2009. 135(1): p. 16-26. 